\section{P18.02 CIRCUMCISION OF HIV POSITIVE MALES WILL NOT UNDERMINE THE BENEFITS OF VOLUNTARY MEDICAL MALE CIRCUMCISION PROGRAMS IN AFRICA}

${ }^{1}$ Susanne F Awad, ${ }^{2,3}$ Sema K Sgaier, ${ }^{2}$ Fiona K Lau, ${ }^{1}$ Yousra A Mohamoud, ${ }^{4}$ Bushimbwa C Tambatamba, ${ }^{5}$ Katharine E Kripke, ${ }^{6}$ Anne G Thomas, ${ }^{7}$ Naomi Bock, ${ }^{8}$ Jason B Reed, ${ }^{9}$ Emmanuel Njeuhmeli, ${ }^{1,10,11}$ Laith J Abu-Raddad*. 'Infectious Disease Epidemiology Group, Weill Cornell Medical College - Qatar, Cornell University, Qatar Foundation - Education City, Doha, Qatar; ${ }^{2}$ Integrated Delivery, Global Development Program, Bill \& Melinda Gates Foundation, Seattle, Washington, USA; ${ }^{3}$ Department of Global Health, University of Washington, Seattle, Washington, USA; ${ }^{4}$ Ministry of Community Development and Mother and Child Health, Lusaka, Zambia; ${ }^{5}$ Health Policy Initiative, Avenir Health, Washington, District of Columbia, USA; ${ }^{6}$ Naval Health Research Center, US Department of Defense, San Diego, California, USA; ${ }^{7}$ Division of Global HIVIAIDS, Center for Global Health, Centers for Disease Control and Prevention, Atlanta, Georgia, USA; ${ }^{8}$ Office of the US Global AIDS Coordinator, Washington, District of Columbia, USA; ${ }^{9}$ United States Agency for International Development, Washington, District of Columbia, USA; ${ }^{10}$ Department of Healthcare Policy and Research, Weill Cornell Medical College, Cornell University, New York, New York, USA; ${ }^{11}$ Vaccine and Infectious Disease Division, Fred Hutchinson Cancer Research Center, Seattle, Washington, USA

\section{$10.1136 /$ sextrans-2015-052270.625}

Introduction The epidemiological and programmatic implications of inclusivity of HIV positive males in voluntary medical male circumcision (VMMC) programs are uncertain. We modelled these implications in Zambia as an illustrative example.

Methods We used the Age-Structured Mathematical (ASM) model to evaluate the effectiveness (number of VMMCs needed to avert one HIV infection) of VMMC scale-up scenarios with varying proportions of HIV positive males. The range of proportions across the scenarios was derived from empirical data and programmatic considerations.

Results The number of VMMCs needed to avert one HIV infection in Zambia was projected to increase from 12.2 VMMCs per HIV infection averted, in a program that circumcises only HIV negative males, to 14.0 in a program that includes HIV positive males, based on their prevalence in the population. However, if a program that only reaches out to negative males is associated with $20 \%$ lower uptake among higher-risk males, the effectiveness would be 13.2 VMMCs per infection averted. If improved inclusivity of positive males is associated with $20 \%$ higher uptake among higher-risk males, the effectiveness would be 12.4. As the assumed VMMC efficacy against male-to-female HIV transmission is increased from $0 \%$ to $20 \%$ and $46 \%$, the effectiveness of circumcising regardless of HIV status improves from 14.0 to 11.5 and 9.1, respectively. HIV incidence rate reduction among females would increase accordingly from $24.7 \%$ to $34.8 \%$ and $50.4 \%$, respectively.

Conclusion Improving inclusivity of males in VMMC programs regardless of HIV status increases VMMC effectiveness, if there is moderate increase in VMMC uptake among higher-risk males and/or if there is moderate efficacy for VMMC against male-tofemale transmission. In these circumstances, VMMC programs can reduce HIV incidence rate in males by nearly as much as expected by some anti-retroviral therapy programs, and additionally, females can benefit from the intervention by nearly as much as males.

Disclosure of interest statement This publication is based on research funded by the Bill and Melinda Gates Foundation. Infrastructure support was provided by the Biostatistics, Epidemiology, and Biomathematics Research Core at the Weill Cornell Medical College in Qatar. The content of this manuscript is the sole responsibility of the authors. The information provided here is not official US Government information and does not necessarily represent the views or positions of United States Agency for International Development, the United States Government, or the Bill and Melinda Gates Foundation.

\section{P18.03 RAPID TRAINING AND IMPLEMENTATION OF THE POLLOCK TECHNIQUE, A SAFE, RAPID AND EFFECTIVE NEWBORN CIRCUMCISION PROCEDURE, IN A LOW- RESOURCE SETTING}

${ }^{1} \mathrm{~N}$ Kojima* ${ }^{2}{ }^{2} \mathrm{CC}$ Bristow, ${ }^{3} \mathrm{~N}$ Pollock, ${ }^{4} \mathrm{P}$ Crouse, ${ }^{5} \mathrm{H}$ Theodore, ${ }^{5} \mathrm{~J}$ Bonhomme, ${ }^{5} \mathrm{CF}$ Gaston, ${ }^{6} \mathrm{JG}$ Dévieux, ${ }^{5,7} \mathrm{JW}$ Pape, ${ }^{2,8} \mathrm{JD}$ Klausner. 'David Geffen School of Medicine, University of California Los Angeles, USA; ${ }^{2}$ Department of Epidemiology, Fielding School of Public Health, University of California Los Angeles, USA; ${ }^{3}$ Department of Medicine, University of British Columbia, Canada; ${ }^{4}$ ntramed Medical Centre, Canada; ${ }^{5}$ Les Centres GHESKIO, Haiti; ${ }^{6}$ Robert Stempel School of Public Health, Florida International University, USA; ${ }^{7}$ Division of Infectious Disease and International Medicine, Weill Medical College of Cornell University, USA; ${ }^{8}$ Departments of Medicine, David Geffen School of Medicine, University of California Los Angeles, USA

\subsection{6/sextrans-2015-052270.626}

Background Male circumcision is highly protective against urinary tract infections, inflammatory conditions of the penis, sexually transmitted infections, and urogenital cancers. We aimed to re-introduce newborn male circumcision through the creation of a training program in Port-au-Prince, Haiti-an area with a considerable burden of preventable urogenital infections, sexually transmitted infections, and low circumcision rate-after an earlier study reported that a majority of Haitian medical providers were in need of and wanted newborn circumcision training.

Methods The rapid, newborn circumcision training program was conducted in November 2014 at the GHESKIO Health Centres, a large, non-governmental clinic offering comprehensive paediatric and adult health services. Two Haitian obstetricians and seven nurses learned administrative, educational, and clinical newborn male circumcision procedures. Complications were monitored and surveys were given to the obstetricians, nurses, and parents of the newborns for assessment.

Results Upon training completion, one of two obstetricians achieved procedural competence. The team circumcised 92 newborns with minor bleeding observed in 1 infant; there were 0 moderate or severe complications. Obstetricians, nurses, and parents of the newborns reported that they were very satisfied with the program $(100 \%, 92 \%$, and 94\%, respectively). Of the parents, $100 \%$ reported they would recommend the procedure to their friends and $100 \%$ reported that they were satisfied with the cosmetic result of the procedure. The nurse-collected survey indicated that $100 \%$ of the procedures followed the proper protocol. Since the rapid newborn circumcision training program concluded, the GHESKIO Health Centres have dedicated two days a week to perform newborn male circumcisions, averaging 14 patients per week

Conclusion Introduction of a newborn male circumcision training program was feasible, achieving an acceptable rate of procedural competency and high quality services. Permanent resources now exist in Haiti to train additional providers to perform newborn male circumcisions.

Funding UCLA Centre for AIDS Research (CFAR) NIH/NIAID AI028697, NIH/NICHD R21HD076685, Pollock Foundation, and Klausner Research and Education Fund. 